\title{
ON MONOCHROMATIC ASCENDING WAVES
}

\author{
Tim LeSaulnier ${ }^{1}$ \\ and \\ Aaron Robertson \\ Department of Mathematics, Colgate University, Hamilton, NY 13346 \\ aaron@math. colgate.edu
}

\begin{abstract}
A sequence of positive integers $w_{1}, w_{2}, \ldots, w_{n}$ is called an ascending wave if $w_{i+1}-$ $w_{i} \geq w_{i}-w_{i-1}$ for $2 \leq i \leq n-1$. For integers $k, r \geq 1$, let $A W(k ; r)$ be the least positive integer such that under any $r$-coloring of $[1, A W(k ; r)]$ there exists a $k$-term monochromatic ascending wave. The existence of $A W(k ; r)$ is guaranteed by van der Waerden's theorem on arithmetic progressions since an arithmetic progression is, itself, an ascending wave. Originally, Brown, Erdős, and Freedman defined such sequences and proved that $k^{2}-k+1 \leq A W(k ; 2) \leq \frac{1}{3}\left(k^{3}-4 k+9\right)$. Alon and Spencer then showed that $A W(k ; 2)=\Theta\left(k^{3}\right)$. In this article, we show that $A W(k ; 3)=\Theta\left(k^{5}\right)$ as well as offer a proof of the existence of $A W(k ; r)$ independent of van der Waerden's theorem. Furthermore, we prove that for any $\epsilon>0$ and any fixed $r \geq 1$,

$$
\frac{k^{2 r-1-\epsilon}}{2^{r-1}(40 r)^{r^{2}-1}}(1+o(1)) \leq A W(k ; r) \leq \frac{k^{2 r-1}}{(2 r-1) !}(1+o(1))
$$

which, in particular, improves upon the best known upper bound for $A W(k ; 2)$. Additionally, we show that for fixed $k \geq 3$,

$$
A W(k ; r) \leq \frac{2^{k-2}}{(k-1) !} r^{k-1}(1+o(1))
$$
\end{abstract}

\section{Introduction}

A sequence of positive integers $w_{1}, w_{2}, \ldots, w_{n}$ is called an ascending wave if $w_{i+1}-w_{i} \geq$ $w_{i}-w_{i-1}$ for $2 \leq i \leq n-1$. For $k, r \in \mathbb{Z}^{+}$, let $A W(k ; r)$ be the least positive integer such that under any $r$-coloring of $[1, A W(k ; r)]$ there exists a monochromatic $k$-term ascending wave. Although guaranteed by van der Waerden's theorem, the existence of $A W(k ; r)$ can be proven independently, as we will show.

Bounds on $A W(k ; 2)$ have appeared in the literature. Brown, Erdös, and Freedman [2] showed that for all $k \geq 1$,

$$
k^{2}-k+1 \leq A W(k ; 2) \leq \frac{k^{3}}{3}-\frac{4 k}{3}+3 .
$$

\footnotetext{
${ }^{1}$ This work was done as part of a high honor thesis in mathematics while the first author was an undergraduate at Colgate University, under the directorship of the second author.
} 
Soon after, Alon and Spencer [1] showed that for sufficiently large $k$,

$$
A W(k ; 2)>\frac{k^{3}}{10^{21}}-\frac{k^{2}}{10^{20}}-\frac{k}{10}+4 .
$$

Recently, Landman and Robertson [4] proposed the refinement of the bounds on $A W(k ; 2)$ and the study of $A W(k ; r)$ for $r \geq 3$. (Note: Since [4] concerns descending waves, we remark that in any finite interval, descending waves are ascending waves when we transverse the interval from right to left.) Here, we offer bounds on $A W(k ; r)$ for all $r \geq 1$, improving upon the previous upper bound for $A W(k ; 2)$.

\section{An Upper Bound}

To show that $A W(k ; r) \leq \Theta\left(k^{2 r-1}\right)$ is straightforward. We will first show that $A W(k ; r) \leq$ $k^{2 r-1}$ by induction on $r$. The case $r=1$ is trivial; for $r \geq 2$, assume $A W(k ; r-1) \leq k^{2 r-3}$ and consider any $r$-coloring of $\left[1, k^{2 r-1}\right]$. Set $w_{1}=1$ and let the color of 1 be red. In order to avoid a monochromatic $k$-term ascending wave there must exist an integer $w_{2} \in\left[2, k^{2 r-3}+1\right]$ that is colored red, lest the inductive hypothesis guarantee a $k$-term monochromatic ascending wave of some color other than red (and we are done). Similarly, there must be an integer $w_{3} \in$ $\left[w_{2}+\left(w_{2}-w_{1}\right), w_{2}+\left(w_{2}-w_{1}\right)+k^{2 r-3}-1\right]$ that is colored red to avoid a monochromatic $k$-term ascending wave. Iterating this argument defines a monochromatic (red) $k$-term ascending wave $w_{1}, w_{2}, \ldots, w_{k}$, provided that $w_{k} \leq k^{2 r-1}$. Since for $i \geq 2, w_{i+1} \leq w_{i}+\left(w_{i}-w_{i-1}\right)+k^{2 r-3}$ we see that $w_{i+1}-w_{i} \leq i k^{2 r-3}$ for $i \geq 1$. Hence, $w_{k}-w_{1}=\sum_{i=1}^{k-1}\left(w_{i+1}-w_{i}\right) \leq \sum_{i=1}^{k-1} i k^{2 r-3} \leq$ $k^{2 r-1}-1$ and we are done.

In this section we provide a better upper bound. Our main theorem in this section follows.

Theorem 1.1 For fixed $r \geq 1$,

$$
A W(k ; r) \leq \frac{k^{2 r-1}}{(2 r-1) !}(1+o(1)) .
$$

We will prove Theorem 1.1 via a series of lemmas, but first we introduce some pertinent notation.

Notation For $k \geq 2$ and $M \geq A W(k ; r)$, let $\Psi^{M}(r)$ be the collection of all $r$-colorings of $[1, M]$. For $\psi \in \Psi^{M}(r)$, let $\chi_{k}(\psi)$ be the set of all monochromatic $k$-term ascending waves under $\psi$. For each monochromatic $k$-term ascending wave $w=\left\{w_{1}, w_{2}, \ldots, w_{k}\right\} \in \chi_{k}(\psi)$, define the $i^{\text {th }}$ difference, $d_{i}(w)=w_{i+1}-w_{i}$, for $1 \leq i \leq k-1$. For $\psi \in \Psi^{M}(r)$, define

$$
\delta_{k}(\psi)=\min \left\{d_{k-1}(w) \mid w \in \chi_{k}(\psi)\right\},
$$

i.e., the minimum last difference over all monochromatic $k$-term ascending waves under $\psi$. Lastly, define

$$
\Delta_{k, r}^{M}=\max \left\{\delta_{k}(\psi) \mid \psi \in \Psi^{M}(r)\right\}
$$


These concepts will provide us with the necessary tools to prove Theorem 1.1.

We begin with an upper bound for $A W(k ; r)$, which is the recusively defined function in the following definition.

Definition 1.2 For $k, r \geq 1$, let $M(k ; 1)=k, M(1 ; r)=1, M(2 ; r)=r+1$, and define, for $k \geq 3$ and $r \geq 2$,

$$
M(k ; r)=M(k-1 ; r)+\Delta_{k-1, r}^{M(k-1 ; r)}+M(k ; r-1)-1 .
$$

Using this definition, we have the following result.

Lemma 1.3 For all $k, r \geq 1, A W(k ; r) \leq M(k ; r)$.

Proof. Noting that the cases $k+r=2,3$, and 4 are, by definition, true, we proceed by induction on $k+r$ using $k+r=5$ as our basis. We have $M(3 ; 2)=7$. An easy calculation shows that $A W(3 ; 2)=7$. So, for some $n \geq 5$, we assume Lemma 1.1 holds for all $k, r \geq 1$ such that $k+r=n$. Now, consider $k+r=n+1$. The result is trivial when $k=1$ or 2 , or if $r=1$, thus we may assume $k \geq 3$ and $r \geq 2$. Let $\psi$ be an $r$-coloring of $[1, M(k ; r)]$. We will show that $\psi$ admits a monochromatic $k$-term ascending wave, thereby proving Lemma 1.3.

By the inductive hypothesis, under $\psi$ there must be a monochromatic $(k-1)$-term ascending wave $w=\left\{w_{1}, w_{2}, \ldots, w_{k-1}\right\} \subseteq[1, M(k-1 ; r)]$ with $d_{k-2}(w) \leq \Delta_{k-1, r}^{M(k-1 ; r)}$. Let

$$
N=\left[w_{k-1}+\Delta_{k-1, r}^{M(k-1 ; r)}, w_{k-1}+\Delta_{k-1, r}^{M(k-1 ; r)}+M(k ; r-1)-1\right] .
$$

If there exists $q \in N$ colored identically to $w$, then $w \cup\{q\}$ is a monochromatic $k$-term ascending wave, since $q-w_{k-1} \geq \Delta_{k-1, r}^{M(k-1 ; r)} \geq d_{k-2}(w)$. If there is no such $q \in N$, then $N$ contains integers of at most $r-1$ colors. Since $|N|=M(k ; r-1)$, the inductive hypothesis guarantees that we have a monochromatic $k$-term ascending wave in $N$. As

$$
w_{k-1}+\Delta_{k-1, r}^{M(k-1 ; r)}+M(k ; r-1)-1 \leq M(k ; r),
$$

the proof is complete.

We now proceed to bound $M(k ; r)$. We start with the following lemma.

Lemma 1.4 Let $k \geq 3$ and $r \geq 2$. Let $M(k ; r)$ be as in Definition 1.2. Then

$$
\Delta_{k, r}^{M(k ; r)} \leq \Delta_{k-1, r}^{M(k-1 ; r)}+M(k ; r-1)-1 .
$$

Proof. Let $\psi, w$, and $N$ be as defined in the proof of Lemma 1.3. If there exists $q \in N$ colored identically to $w$, then

$$
\delta_{k}(\psi) \leq d_{k-1}(w \cup\{q\}) \leq \Delta_{k-1, r}^{M(k-1 ; r)}+M(k ; r-1)-1 .
$$


If there is no such $q \in N$, then there exists a monochromatic $k$-term ascending wave, say $v$, in $N$. Hence, $\delta_{k}(\psi) \leq d_{k-1}(v) \leq M(k ; r-1)-(k-1)$. Since $\psi$ was chosen arbitrarily, it follows that

$$
\Delta_{k, r}^{M(k ; r)} \leq \Delta_{k-1, r}^{M(k-1 ; r)}+M(k ; r-1)-1 .
$$

The following lemma will provide a means for recursively bounding $M(k ; r)$.

Lemma 1.5 Let $k \geq 3$ and $r \geq 2$. Let $M(k ; r)$ be as in Definition 1.2. Then

$$
M(k ; r) \leq \sum_{i=0}^{k-3}((i+1) M(k-i ; r-1))-\frac{k^{2}}{2}+\frac{3 k}{2}+(k-1) r
$$

Proof. We proceed by induction on $k$. Consider $M(3 ; r)$. We have

$$
M(3 ; r)=M(2 ; r)+\Delta_{2, r}^{M(2 ; r)}+M(3 ; r-1)-1 .
$$

Since $M(2 ; r)=r+1$ and $\Delta_{2, r}^{M(2 ; r)}=r$, we have $M(3 ; r)=M(3 ; r-1)+2 r$, thereby finishing the case $k=3$ and arbitrary $r$. Now assume that Lemma 1.5 holds for some $k \geq 3$. The inductive hypothesis, along with Lemma 1.4, give us

$$
\begin{aligned}
M(k+1 ; r)= & M(k ; r)+\Delta_{k, r}^{M(k ; r)}+M(k+1 ; r-1)-1 \\
\leq & \sum_{i=0}^{k-3}((i+1) M(k-i ; r-1))-\frac{k^{2}}{2}+\frac{3 k}{2}+(k-1) r \\
& \quad+\Delta_{k, r}^{M(k ; r)}+M(k+1 ; r-1)-1 \\
\leq & \sum_{i=0}^{k-3}((i+1) M(k-i ; r-1))-\frac{k^{2}}{2}+\frac{3 k}{2}+(k-1) r \\
& \quad+\Delta_{2, r}^{M(2 ; r)}+\sum_{i=0}^{k-3} M(k-i ; r-1) \\
& \quad+M(k+1 ; r-1)-(k-2)-1 \\
\leq & \sum_{i=0}^{k-2}((i+1) M(k+1-i ; r-1))-\frac{(k+1)^{2}}{2}+\frac{3(k+1)}{2}+k r
\end{aligned}
$$

as desired.

Now, for $r \geq 2$, an upper bound on $M(k ; r)$ can be obtained by using Lemma 1.5. We offer one additional lemma, from which Theorem 1.1 will follow by application of Lemma 1.3.

Lemma 1.6 For $r \geq 1$, there exists a polynomial $p_{r}(k)$ of degree at most $2 r-2$ such that

$$
M(k ; r) \leq \frac{k^{2 r-1}}{(2 r-1) !}+p_{r}(k)
$$


for all $k \geq 3$.

Proof. We have $M(k ; 1)=k$, so we can take $p_{1}(k)=1$, having degree 0 . We proceed by induction on $r$. Let $r \in \mathbb{Z}^{+}$and assume the lemma holds for $r$. Lemma 1.5 gives

$$
\begin{gathered}
M(k ; r+1) \leq \sum_{j=3}^{k}((k-j+1) M(j ; r))-\frac{k^{2}}{2}+\frac{3 k}{2}+(k-1)(r+1) \\
\leq k \sum_{j=3}^{k}\left(\frac{j^{2 r-1}}{(2 r-1) !}+p_{r}(j)\right)-\sum_{j=3}^{k}\left((j-1)\left(\frac{j^{2 r-1}}{(2 r-1) !}+p_{r}(j)\right)\right) \\
\quad-\frac{k^{2}}{2}+\frac{3 k}{2}+(k-1)(r+1) .
\end{gathered}
$$

By Faulhaber's formula [3], for some polynomial $p_{r+1}(k)$ of degree at most $2 r$, we now have

$$
M(k ; r+1) \leq k \frac{\frac{k^{2 r}}{2 r}}{(2 r-1) !}-\frac{\frac{k^{2 r+1}}{2 r+1}}{(2 r-1) !}+p_{r+1}(k)=\frac{k^{2 r+1}}{(2 r+1) !}+p_{r+1}(k)
$$

and the proof is complete.

Theorem 1.1 now follows from Lemmas 1.3 and 1.6.

Interestingly, Lemma 1.5 can also be used to show the following result.

Theorem 1.7 For fixed $k \geq 3$,

$$
A W(k ; r) \leq \frac{2^{k-2}}{(k-1) !} r^{k-1}(1+o(1))
$$

Proof. In analogy to Lemma 1.6, we show that for $k \geq 3$ and $r \geq 2$, there exists a polynomial $s_{k}(r)$ of degree at most $k-2$ such that

$$
M(k ; r) \leq \frac{2^{k-2}}{(k-1) !} r^{k-1}+s_{k}(r)
$$

We proceed by induction on $k$. Let $r \geq 2$ be arbitrary. By definition we have

$$
M(3 ; r)=M(3 ; r-1)+2 r .
$$

Since $M(3 ; 1)=3$, we get

$$
M(3 ; r)=M(3 ; 1)+\sum_{i=2}^{r} 2 i=r^{2}+r+1,
$$

for $r \geq 2$, which serves as our basis. Now, for given $k \geq 4$, let $\hat{s}_{3}(r)=(k-1) r-\frac{k^{2}}{2}+\frac{3 k}{2}$ and assume (1) holds for all integers $3 \leq j \leq k-1$ and for all $r \geq 2$. Lemma 1.5 yields

$$
\begin{aligned}
M(k ; r) & \leq \sum_{i=0}^{k-3}((i+1) M(k-i ; r-1))+\hat{s}_{3}(r) \\
& =M(k ; r-1)+\sum_{i=1}^{k-3}((i+1) M(k-i ; r-1))+\hat{s}_{3}(r) .
\end{aligned}
$$


Now, by the inductive hypothesis, for $1 \leq i \leq k-3$, we have that

$$
\begin{aligned}
M(k-i ; r-1) & \leq \frac{2^{k-i-2}}{(k-i-1) !}(r-1)^{k-i-1}+s_{k-i}(r-1) \\
& =\frac{2^{k-i-2}}{(k-i-1) !} r^{k-i-1}+\tilde{s}_{k-i}(r)
\end{aligned}
$$

where $\tilde{s}_{k-i}(r)$ is a polynomial of degree at most $k-i-2 \leq k-3$. This gives us that

$$
\begin{aligned}
\sum_{i=1}^{k-3}(i+1) M(k-i ; r-1)+\hat{s}_{3}(r) & \leq \sum_{i=1}^{k-3}\left((i+1)\left(\frac{2^{k-i-2}}{(k-i-1) !} r^{k-i-1}+\tilde{s}_{k-i}(r)\right)\right)+\hat{s}_{3}(r) \\
& =2 \cdot \frac{2^{k-3}}{(k-2) !} r^{k-2}+\check{s}_{k-1}(r),
\end{aligned}
$$

where $\check{s}_{k-1}(r)$ is a polynomial of degree at most $k-3$. Hence, we have

$$
\begin{aligned}
M(k ; r) & \leq M(k ; r-1)+2 \cdot \frac{2^{k-3}}{(k-2) !} r^{k-2}+\check{s}_{k-1}(r) \\
& =M(k ; r-1)+\frac{2^{k-2}}{(k-2) !} r^{k-2}+\check{s}_{k-1}(r) .
\end{aligned}
$$

As $M(k ; 1)=k$, we have a recursive bound on $M(k ; r)$ for $r \geq 2$. Faulhaber's formula [3] yields

$$
M(k ; r) \leq M(k ; 1)+\sum_{i=2}^{r}\left(\frac{2^{k-2}}{(k-2) !} i^{k-2}+\check{s}_{k-1}(r)\right) \leq \frac{2^{k-2}}{(k-1) !} r^{k-1}+s_{k}(r),
$$

where $s_{k}(r)$ is a polynomial of degree at most $k-2$. By Lemma 1.3, the result follows.

\section{A Lower Bound for more than Three Colors}

We now provide a lower bound on $A W(k ; r)$ for arbitrary fixed $r \geq 1$. We generalize an argument of Alon and Spencer [1] to provide our lower bound.

We will use $\log x=\log _{2} x$ throughout. Also, by $k=x$ for $x \notin \mathbb{Z}^{+}$we mean $k=\lfloor x\rfloor$.

We proceed by defining a certain type of random coloring. To this end, let $r \geq 2$ and consider the $r \times 2 r$ matrix $A_{0}=\left(a_{i j}\right)$ :

$$
\left[\begin{array}{ccccccccc}
0 & 0 & 1 & 1 & 2 & 2 & \ldots & (r-1) & (r-1) \\
0 & 1 & 1 & 2 & 2 & 3 & \ldots & (r-1) & 0 \\
0 & 2 & 1 & 3 & 2 & 4 & \ldots & (r-1) & 1 \\
\vdots & & & & & \vdots & & \vdots & \vdots \\
0 & (r-1) & 1 & 0 & 2 & 1 & \ldots & (r-1) & (r-2)
\end{array}\right]
$$


i.e., for $j \in[0, r-1]$, we have $a_{i, 2 j+1}=j$, for all $1 \leq i \leq r$, and $a_{i, 2 j+2} \equiv i+j-1(\bmod r)$.

Next, we define $A_{j}=A_{0} \oplus \mathbf{j}$ where $\oplus$ means that entry-wise addition is done modulo $r$ and $\mathbf{j}$ is the $r \times 2 r$ matrix with all entries equal to $j$.

Consider the $r^{2} \times 2 r$ matrix $A=\left[\begin{array}{lllll}A_{0} & A_{1} & A_{2} & \ldots & A_{r-1}\end{array}\right]^{t}$.

In the sequel, we will use the following notation.

Notation For $k, r \geq 1$, let

$$
N_{r}=\frac{1}{2^{r-1}(40 r)^{r^{2}-1}} \quad \text { and } \quad b=A W\left(\frac{k}{10(4 r-4)} ; r-1\right)-1 .
$$

Furthermore, let $\gamma_{i}$ be an $(r-1)$-coloring of $[1, b]$ with no monochromatic $\frac{k}{10(4 r-4)}$-term ascending wave, where the $r-1$ colors used are $\{0,1, \ldots, i-1, i+1, i+2, \ldots, r-1\}$ (i.e., color $i$ is not used, and hence the subscript on $\gamma$ ).

Fix $\epsilon>0$. We next describe how we randomly $r$-color $\left[1, M_{\epsilon}\right]$, where

$$
M_{\epsilon}=N_{r} k^{2 r-1-\epsilon} \text {. }
$$

We partition the interval $\left[1, M_{\epsilon}\right]$ into consecutive intervals of length $b$ and denote the $i^{\text {th }}$ such interval by $B_{i}$ and call it a block (note that the last block may be a block of length less than $b)$. For $i=1,2, \ldots,\left\lceil\frac{M_{\epsilon}}{2 r b}\right\rceil$, let

$$
C_{i}=\bigcup_{j=1}^{2 r} B_{2 r(i-1)+j} .
$$

For each $C_{i}$, we randomly choose a row in $A$, say $\left(s_{1}, s_{2}, \ldots, s_{2 r}\right)$. We color the $j^{\text {th }}$ block of $C_{i}$ by $\gamma_{s_{j}}$. By $\operatorname{col}\left(B_{i}\right)$ we mean the coloring of the $i^{\text {th }}$ block, $1 \leq i \leq\left\lceil\frac{M_{\epsilon}}{b}\right\rceil$, which is one of $\gamma_{0}, \gamma_{1}, \ldots, \gamma_{r-1}$. In the case when $2 r \cdot\left\lceil\frac{M_{\epsilon}}{2 r b}\right\rceil \neq\left\lceil\frac{M_{\epsilon}}{b}\right\rceil$, the $j^{\text {th }}$ block (and block of length less than $b$, if present) of $C_{\left\lceil\frac{M_{\epsilon}}{2 r b}\right\rceil}$ is colored by $\gamma_{s_{j}}$ for all possible $j$ (so that the entries in the row of $A$ chosen for $C_{\left\lceil\frac{M_{\epsilon}}{2 r b}\right\rceil}$ may not all be used).

The following is immediate by construction.

\section{Lemma 2.1}

(i) For all $1 \leq i \leq 2 r b, P\left(\operatorname{col}\left(B_{i}\right)=\gamma_{c}\right)=\frac{1}{r}$ for each $c=0,1, \ldots, r-1$.

(ii) For any $i, P\left(\operatorname{col}\left(B_{i}\right)=\gamma_{c}\right.$ and $\left.\operatorname{col}\left(B_{i+1}\right)=\gamma_{d}\right)=\frac{1}{r^{2}}$ for any $c$ and $d$.

(iii) The colorings of blocks with at least $2 r$ other blocks between them are mutually independent.

The approach we take, following Alon and Spencer [1], is to show that there exists a coloring such that for any monochromatic $\frac{k}{2}$-term ascending wave $w_{1}, w_{2}, \ldots, w_{k / 2}$ we have 
$w_{k / 2}-w_{k / 2-1} \geq \delta k^{2 r-2-\epsilon / 2}$ for some $\delta>0$. The following definition and lemma, which are generalizations of those found in [1], will give us the desired result.

Definition 2.2 An arithmetic progression $x_{1}<x_{2}<\cdots<x_{t}$ is called a good progression if for each $c \in\{0,1, \ldots, r-1\}$, there exist $i$ and $j$ such that $x_{i} \in B_{j}$ and $\operatorname{col}\left(B_{j}\right)=\operatorname{col}\left(B_{j+1}\right)=$ $\gamma_{c}$. An arithmetic progression that is not good is called a bad progression.

Lemma 2.3 For $k, r \geq 2$, let $t=\frac{(4 r-2)(2 r+1)}{\log \left(r^{2} /\left(r^{2}-1\right)\right)} \log k+\frac{(2 r+1)(\log r+1)}{\log \left(r^{2} /\left(r^{2}-1\right)\right)}$. For $k$ sufficiently large, the probability that there is a bad progression in a random coloring of $\left[1, M_{\epsilon}\right]$ with difference greater than $b$, of $t$ terms, is at most $\frac{1}{2}$.

Proof. Let $x_{1}<x_{2}<\cdots<x_{t}$ be a progression with $x_{2}-x_{1}>b$. Then no 2 elements belong to the same block. For each $i, 1 \leq i \leq \frac{t}{2 r+1}$, let $D_{i}$ be the block in which $x_{(2 r+1) i}$ resides, and let $E_{i}$ be the block immediately following $D_{i}$. Then, the probability that the progression is bad is at most

$$
p=\sum_{j=1}^{r} P\left(\nexists i \in\left[1, \frac{t}{2 r+1}\right]: \operatorname{col}\left(D_{i}\right)=\operatorname{col}\left(E_{i}\right)=\gamma_{j}\right)
$$

We have

$$
\begin{aligned}
p & \leq r P\left(\nexists i \in\left[1, \frac{t}{2 r+1}\right]: \operatorname{col}\left(D_{i}\right)=\operatorname{col}\left(E_{i}\right)=\gamma_{0}\right) \\
& =r\left(\frac{r^{2}-1}{r^{2}}\right)^{\frac{t}{2 r+1}} \\
& \leq r\left(\frac{r^{2}-1}{r^{2}}\right)^{\frac{(4 r-2)}{\log \left(r^{2} /\left(r^{2}-1\right)\right)} \log k+\frac{\log r+1}{\log \left(r^{2} /\left(r^{2}-1\right)\right)}} \\
& \leq \frac{2^{-1}}{k^{4 r-2}}
\end{aligned}
$$

for $k$ sufficiently large.

Since the number of $t$-term arithmetic progressions in $\left[1, M_{\epsilon}\right]$ is less than $M_{\epsilon}^{2}<k^{4 r-2}$, the probability that there is a bad progression is less than

$$
k^{4 r-2} \cdot \frac{2^{-1}}{k^{4 r-2}}=\frac{1}{2}
$$

thereby completing the proof.

Lemma 2.4 Consider any $r$-coloring of $\left[1, M_{\epsilon}\right]$ having no bad progression with difference greater than $b$ of $t$ terms ( $t$ from Lemma 2.3). Then, for any $\epsilon>0$, for $k$ sufficiently large, any monochromatic $\frac{k}{2}$-term ascending wave $w_{1}, w_{2}, \ldots, w_{k / 2}$ has $w_{k / 2}-w_{k / 2-1} \geq b k^{1-\epsilon / 2}=$ $\Theta\left(k^{2 r-2-\epsilon / 2}\right)$.

Proof. At most $4 r-4$ consecutive blocks can have a specific color in all of them. (To achieve this, say the color is 0 . The random coloring must have chosen row 1 followed by row $r+1$, to have $\gamma_{0} \gamma_{0} \gamma_{1} \gamma_{1} \cdots \gamma_{r-1} \gamma_{r-1} \gamma_{1} \gamma_{1} \gamma_{2} \gamma_{2} \cdots \gamma_{0} \gamma_{0}$.) Since each block has a monochromatic 
ascending wave of length at most $\frac{k}{10(4 r-4)}-1$, any $4 r-4$ consecutive blocks contribute less than $\frac{k}{10}$ terms to a monochromatic ascending wave. After that, the next difference must be more than $b$.

Let $Z=a_{1}, a_{2}, \ldots, a_{k / 2}$ be monochromatic ascending wave under our random coloring. Then, there exists $i<\frac{k}{10}$ such that $a_{i+1}-a_{i} \geq b+1$. Now let $X=x_{1}, x_{2}, \ldots, x_{t}$ be a $t$-term good progression with $x_{1}=a_{i}$ and $d=x_{2}-x_{1}=a_{i+1}-a_{i} \geq b+1$.

Assume, without loss of generality, that the color of $Z$ is 0 . Since $X$ is a good progression, there exists $x_{j} \in B_{\ell}$ with $\operatorname{col}\left(B_{\ell}\right)=\operatorname{col}\left(B_{\ell+1}\right)=\gamma_{0}$ for some block $B_{\ell}$. Since $a_{i+j} \geq x_{j}$ as $Z$ is an ascending wave, we see that $a_{i+j}-a_{i} \geq j d+b+1$. We conclude that $a_{i+t}-a_{i} \geq t d+b+1$ so that $a_{i+t+1}-a_{i+t} \geq d+\frac{b+1}{t}$. Now, redefine $X=x_{1}, x_{2}, \ldots, x_{t}$ to be the $t$-term good progression with $x_{1}=a_{i+t}$ and $d^{\prime}=x_{2}-x_{1}=a_{i+t+1}-a_{i+t} \geq d+\frac{b+1}{t} \geq(b+1)\left(1+\frac{1}{t}\right)$. Repeating the above argument, we see that $a_{i+2 t}-a_{i+t} \geq t d^{\prime}+b+1$ so that $a_{i+2 t}-a_{i+2 t-1} \geq$ $d^{\prime}+\frac{b+1}{t} \geq(b+1)\left(1+\frac{2}{t}\right)$. In general,

$$
a_{i+s t}-a_{i+s t-1} \geq(b+1)\left(1+\frac{s}{t}\right)
$$

for $s=1,2, \ldots \frac{2 k-5 t}{5 t}$. Thus, we have (with $s=\left(k^{1-\epsilon / 2}-1\right) t \leq \frac{2 k-5 t}{5 t}$ for $k$ sufficiently large)

$$
a_{k / 2}-a_{k / 2-1} \geq(b+1)\left(1+\frac{\left(k^{1-\epsilon / 2}-1\right) t}{t}\right)=(b+1) k^{1-\epsilon / 2} .
$$

We are now in a position to state and prove this section's main result.

Theorem 2.5 For fixed $r \geq 1$ and any $\epsilon>0$, for $k$ sufficiently large,

$$
A W(k ; r) \geq \frac{k^{2 r-1-\epsilon}}{2^{r-1}(40 r)^{r^{2}-1}} .
$$

Proof. Fix $\epsilon>0$ and let $M_{\epsilon}=N_{r} k^{2 r-1-\epsilon}$ for $r \geq 1$. We use induction on $r$, with $r=1$ being trivial (since $A W(k ; 1)=k$ ) and $r=2$ following from Alon and Spencer's result [1]. Hence, assume $r \geq 3$ and assume the theorem holds for $r-1$. Using Lemma 2.4, there exists an $r$-coloring $\chi$ of $\left[1, M_{\epsilon}\right]$ such that any monochromatic $\frac{k}{2}$-term ascending wave has last difference at least $(b+1) k^{1-\epsilon / 2}$. This implies that the last term of any monochromatic $k$-term ascending wave under $\chi$ must be at least $\frac{k}{2}+(b+1) k^{1-\epsilon / 2} \cdot \frac{k}{2}>\frac{1}{2}(b+1) k^{2-\epsilon / 2}$.

We have, by the inductive hypothesis and the definition of $b$,

$$
b+1 \geq N_{r-1} \frac{k^{2 r-3-\epsilon / 2}}{40^{2 r-3-\epsilon / 2}(r-1)^{2 r-3-\epsilon / 2}} \geq N_{r-1} \frac{k^{2 r-3-\epsilon / 2}}{40^{2 r-1} r^{2 r-1}} .
$$

Hence, for $k$ sufficiently large, the last term of any monochromatic $k$-term ascending wave under $\chi$ must be greater than

$$
N_{r-1} \cdot \frac{1}{40^{2 r-1} r^{2 r-1}} k^{2 r-3-\epsilon / 2} \cdot \frac{k^{2-\epsilon / 2}}{2}=N_{r} k^{2 r-1-\epsilon}=M_{\epsilon} .
$$


Hence, we have an $r$-coloring of $\left[1, M_{\epsilon}\right]$ with no $k$-term monochromatic ascending wave, for $k$ sufficiently large.

\section{A Lower Bound for Three Colors}

We believe that $A W(k ; r)=\Theta\left(k^{2 r-1}\right)$, however, we have thus far been unable to prove this. The approach of Alon and Spencer [1], which is to show that there exists an $r$-coloring (under a random coloring scheme) such that every monochromatic $\frac{3 k}{4}$-term ascending wave has $d_{3 k / 4-1}>c k^{2 r-2}$ does not work for an arbitrary number of colors with our generalization. However, for 3 colors, we can refine their argument to prove that $A W(k ; 3)=\Theta\left(k^{5}\right)$.

\section{Theorem 3.1}

$$
\frac{k^{5}}{2^{13} \cdot 10^{39}} \leq A W(k ; 3) \leq \frac{k^{5}}{120}(1+o(1))
$$

The upper bound comes from Theorem 1.1, hence we need only prove the lower bound. We use the same coloring scheme as in Section 2 and proceed with a series of lemmas.

Definition 3.2 We call a sequence $x_{1}, x_{2}, \ldots, x_{n}$ with $x_{2}-x_{1} \geq 1$ an almost ascending wave if, for $2 \leq i \leq n-1$, we have $d_{i}=x_{i+1}-x_{i}$ with $d_{i} \geq d_{i-1}-1$, with equality for at least one such $i$ and with the property that if $d_{i}=d_{i-1}-1$ and $d_{j}=d_{j-1}-1$ with $j>i$ there must exist $s, i<s<j$, such that $d_{s} \geq d_{s-1}+1$.

The upper bound of the following proposition is a slight refinement of a result of Alon and Spencer [1, Lemma 1.7].

Proposition 3.3 Denote by $a w(n)$ the number of ascending waves of length $n$ with first term given and $d_{n-1}<\frac{n}{10^{14}}$. Analogously, let $a a w(n)$ be the number of almost ascending waves of length $n$ with first term given and $d_{n-1} \leq \frac{n}{10^{14}}$. Then, for all $n$ sufficiently large,

$$
2^{\frac{n}{2}-1}<a w(n)+a a w(n) \leq 2^{\frac{13 n}{25}} \cdot\left(\frac{3}{2}\right)^{n / 100} .
$$

Proof. We start with the lower bound by constructing a sequence of differences that contribute to either $a w(n)$ or $a a w(n)$. We start by constructing a sequence where all of $\frac{n}{2}-1$ slots contain 2 terms of a sequence. From a list of $\frac{n}{2}-1$ empty slots, choose $j, 0 \leq j \leq \frac{n}{2}-1$, of them. In these slots place the pair $-1,1$. In the remaining slots put the pair 0,0 . We now have a sequence of length $n-2$ or $n-3$. If the length is $n-2$, put a 2 at the end; if the length is $n-3$, put 2,2 at the end. We now have, for each $j$ and each choice of $j$ slots, a distinct sequence of length $n-1$. Denote one such sequence by $s_{1}, s_{2}, \ldots, s_{n-1}$. Using this sequence, we define a sequence of difference $\left\{d_{i}\right\}$ that will correspond to either an ascending wave or an almost ascending wave. To this end, let $d_{1}=1$ and $d_{i}=d_{i-1}+s_{i-1}$ for $i=2,3, \ldots, n$. Since we have the first term of an almost ascending, or ascending, wave $w_{1}, \ldots, w_{n}$ given, such a 
wave is determined by its sequence of differences $w_{i+1}-w_{i}$. Above, we have constructed a sequence $\left\{d_{i}\right\}$ of differences that adhere to the rules of an almost ascending, or ascending, wave. Hence, $a w(n ; r)+a a w(n ; r)>\sum_{j=0}^{\frac{n}{2}-1}\left(\begin{array}{c}\frac{n}{2}-1 \\ j\end{array}\right)=2^{\frac{n}{2}-1}$.

For the upper bound, we follow the proof of Alon and Spencer [1, Lemma 1.7], improving the bound enough to serve our purpose. Their lemma includes the term $\left(\begin{array}{c}n+\left\lceil 10^{-6} n\right\rceil-1 \\ n-1\end{array}\right)$ which we will work on to refine their upper bound on $a w(n)+a a w(n)$.

First, we have

$$
\left(\begin{array}{c}
n+\left\lceil 10^{-6} n\right\rceil-1 \\
n-1
\end{array}\right) \leq\left(\begin{array}{c}
\left(1+10^{-5}\right) n \\
n
\end{array}\right)
$$

for $n$ sufficiently large.

Let $q=\left(1+10^{-5}\right)^{-1}, m=\frac{n}{q}$, and let $H(x)=-x \log x-(1-x) \log (1-x)$ for $0 \leq x \leq 1$ be the binary entropy function. Then we have ${ }^{2}$

$$
\left(\begin{array}{c}
m \\
q m
\end{array}\right) \leq 2^{m H(q)}
$$

Applying this, we have

$$
H(q)=\frac{1}{1+10^{-5}} \log \left(1+10^{-5}\right)-\frac{10^{-5}}{1+10^{-5}} \log \frac{10^{-5}}{1+10^{-5}}
$$

so that

$$
\begin{aligned}
m H(q) & =\left[\log \left(1+10^{-5}\right)-\frac{1}{10^{5}} \log \frac{10^{-5}}{1+10^{-5}}\right] n \\
& =\left[\frac{1}{10^{5}} \log 10^{5}\left(1+10^{-5}\right)^{10^{5}+1}\right] n \\
& \leq\left[\frac{1}{10^{5}} \log e\left(10^{5}+1\right)\right] n .
\end{aligned}
$$

We proceed by noting that

$$
\left[\frac{\log e\left(10^{5}+1\right)}{10^{5}}\right] n \leq\left[\frac{1}{100} \log \frac{3}{2}\right] n .
$$

Hence, $2^{m H(q)} \leq 2^{\frac{n}{100} \log \frac{3}{2}}=\left(\frac{3}{2}\right)^{\frac{n}{100}}$. Now, using Alon and Spencer's result [1, Lemma 1.7], the result follows.

We are now in a position to prove the fundamental lemma of this section. In the proof we refer to the following definition.

${ }^{2}$ Here's a quick derivation: For all $n \geq 1$, we have $\sqrt{2 \pi n} e^{1 /(12 n+1)}(n / e)^{n} \leq n ! \leq \sqrt{2 \pi n} e^{1 /(12 n)}(n / e)^{n}$ (see [5]). Hence, $\left(\begin{array}{c}m \\ q m\end{array}\right) \leq \frac{c}{\sqrt{m(1-q)}}\left(q^{-q}(1-q)^{-(1-q)}\right)^{m}$ for some positive $c<e^{-2}$ (so that $\frac{c}{\sqrt{m(1-q)}}<1$ for $m$ sufficiently large). Using the base $2 \log$, this gives $\left(\begin{array}{c}m \\ q m\end{array}\right) \leq 2^{m H(q)}$. 
Definition 3.3 Let $a_{1}, \ldots, a_{n}$ be an ascending wave and let $x \in \mathbb{Z}^{+}$. We call $\left\lfloor\frac{a_{1}}{x}\right\rfloor,\left\lfloor\frac{a_{2}}{x}\right\rfloor, \cdots,\left\lfloor\frac{a_{n}}{x}\right\rfloor$ the associated $x$-floor wave.

Lemma 3.4 Let

$$
Q=\frac{k^{5}}{2^{13} \cdot 10^{39}}
$$

and let $b=A W(k / 80 ; 2)-1$. The probability that in a random 3-coloring of $[1, Q]$ there is a monochromatic $\frac{k}{4}$-term ascending wave whose first difference is greater than $6 b(=2 r b)$ and whose last difference is smaller than $\frac{k b}{4 \cdot 10^{14}}=\Theta\left(k^{4}\right)$ is less than $\frac{1}{2}$ for $k$ sufficiently large.

Proof. Let $Y=a_{1}<a_{2}<\cdots<a_{k / 4}$ be an ascending wave and let $\left\lfloor\frac{a_{1}}{b}\right\rfloor<\left\lfloor\frac{a_{2}}{b}\right\rfloor<\cdots<\left\lfloor\frac{a_{k / 4}}{b}\right\rfloor$ be the associated $b$-floor wave. Note that this $b$-floor wave is either an ascending wave or an almost ascending wave with last difference at most $\frac{k / 4}{10^{14}}$. Hence, by Proposition 3.2 , the number of such $b$-floor waves is at most, for $k$ sufficiently large,

$$
k^{2} \cdot 2^{\frac{13 k}{100}} \cdot\left(\frac{3}{2}\right)^{k / 400} \leq 2^{\frac{14 k}{100}} \cdot\left(\frac{3}{2}\right)^{k / 400}
$$

(we have less than $k^{2}$ choices for $\left\lfloor\frac{a_{1}}{b}\right\rfloor$ ).

Note that $Y$ is monochromatic of color, say $c$, only if none of the blocks $B_{\left\lfloor\frac{a_{i}}{b}\right\rfloor}, 1 \leq i \leq \frac{k}{4}$, is colored by $\gamma_{c}$. Note that all of these blocks are at least $6(=2 r)$ blocks from each other. We use Lemma 2.1 to give us that the probability that $Y$ is monochromatic is no more than

$$
3\left(\frac{2}{3}\right)^{k / 4}
$$

Thus, the probability that in a random 3-coloring of $[1, Q]$ we have a monochromatic $\frac{k}{4}$-term ascending wave with last difference less than $\frac{k b}{4 \cdot 10^{14}}$ is at most

$$
3 \cdot 2^{\frac{14 k}{100}} \cdot\left(\frac{2}{3}\right)^{99 k / 400}
$$

We have $3<\left(\frac{3}{2}\right)^{3 k / 400}$ for $k$ sufficiently large, so that the above probability is less than

$$
2^{\frac{14 k}{100}} \cdot\left(\frac{2}{3}\right)^{24 k / 100} .
$$

The above quantity is, in particular, less than $1 / 2$ for $k$ sufficiently large.

To finish proving Theorem 3.1, we apply Lemmas 2.3 and 2.4, as well as Lemma 3.5, to show that, for $k$ sufficiently large, there exists a 3-coloring of $[1, Q]$ such that both of the following hold:

1) Any $\frac{k}{2}$-term monochromatic ascending wave has last difference greater than $6 b(=2 r b)$. 
2) Any $\frac{k}{4}$-term monochromatic ascending wave with first difference greater than $6 b(=2 r b)$ has last difference greater than $\frac{k b}{4 \cdot 10^{14}}$.

Hence, we conclude that there is a 3 -coloring of $[1, Q]$ such that any monochromatic $\frac{3 k}{4}$-term ascending wave has last difference greater than $\frac{k b}{4 \cdot 10^{14}}$, for $k$ sufficiently large. This implies that the last term of a monochromatic $k$-term ascending wave must be at least $\frac{3 k}{4}+\frac{k b}{4 \cdot 10^{14}} \cdot \frac{k}{4}$.

We have $b=A W\left(\frac{k}{10(4 r-4)} ; r-1\right)-1$ with $r=3$. By Alon and Spencer's result [1], this gives us

$$
b \geq \frac{k^{3}}{10^{25} \cdot 8^{3}}
$$

for $k$ sufficiently large.

Hence, for $k$ sufficiently large, the last term of a monochromatic $k$-term ascending wave must be at least

$$
\frac{3 k}{4}+\frac{k^{2}}{4^{2} \cdot 10^{14}} \cdot \frac{k^{3}}{10^{25} \cdot 8^{3}}>\frac{k^{5}}{2^{13} \cdot 10^{39}}=Q .
$$

Since we have the existence of a 3 -coloring of $[1, Q]$ with no monochromatic $k$-term ascending wave, this completes the proof of Theorem 3.1 .

Remark From the lower bound given in Proposition 3.3, it is not possible to show that there exists $c>0$ such that $A W(k ; r) \geq c k^{2 r-1}$ for $r \geq 4$, by using the argument presented in Sections 2 and 3. However, we still make the following conjecture.

Conjecture For all $r \geq 1, A W(k ; r)=\Theta\left(k^{2 r-1}\right)$.

Acknowledgment We thank an anonymous referee for a very detailed, knowledgable, and quick reading.

\section{References}

[1] N. Alon and J. Spencer, Ascending waves, J. Combin. Theory, Series A 52 (1989), 275-287.

[2] T. Brown, P. Erdös, and A. Freedman, Quasi-progressions and descending waves, J. Combin. Theory Series A 53 (1990), 81-95.

[3] J. Conway and R. Guy, The Book of Numbers, Springer-Verlag, New York, p. 106, 1996.

[4] B. Landman and A. Robertson, Ramsey Theory on the Integers, American Math. Society, Providence, RI, 317pp., 2003.

[5] H. Robbins, A remark on Stirling's formula, American Math. Monthly 62 (1955), 26-29. 